\title{
Overseas CPD
}

The WSAVA Continuing Education Program aims to deliver CE for

first-opinion companion animal practitioners in countries where access to high quality CE is limited. Zoe Belshaw, Chair of the WSAVA Continuing Education Committee, reports on 2017 successes.

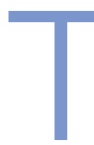

he goal of the WSAVA Continuing Education Program is to provide practical, relevant and inspiring CE that assists general practitioners solve clinical problems regardless of the size and sophistication of their practice. Each country in the CE program is categorized according to its level of need. In the countries where access to CE is most limited the WSAVA supports all the running costs for the CE meeting. When countries are able to support local organizational costs through delegate registration fees and additional local sponsorship, sponsorship is reduced so that only speaker costs are covered. Speakers are drawn from all continents, chosen on the basis of their qualifications and their reputation as being excellent presenters of information. In 2017, the WSAVA CE Africa programme was possible due to the generous support of the WSAVA commercial sponsors Bayer and MSD and association sponsors, BSAVA and NACAM (The Netherlands Association for Companion Animal Medicine) who provided some additional financial support for the meeting in Malawi.

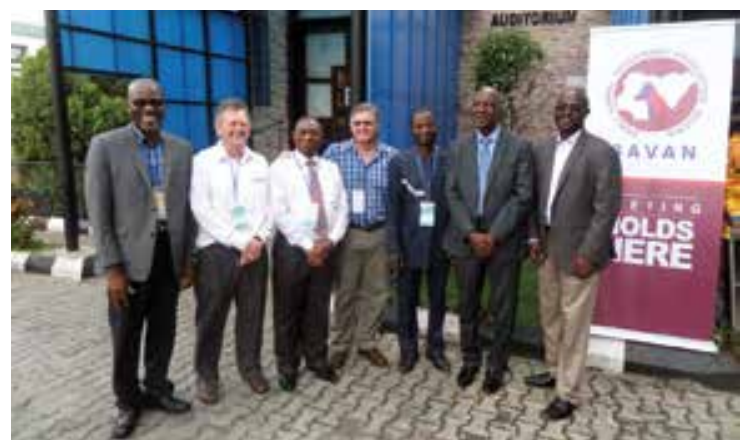

Nigeria's organizing committee with speakers.

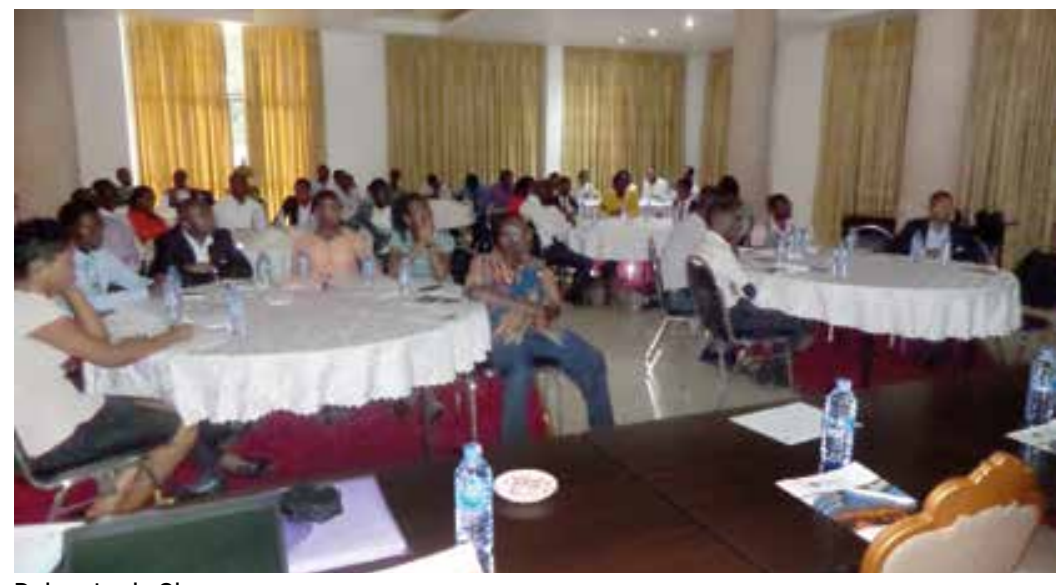

Delegates in Ghana.

In Africa during 2017, CE expanded further to include support for a lecture stream on anaesthesia in Morocco, delivered by Dr Eric Troncy from Montreal, Canada and the first ever meeting in Nigeria on infectious diseases and diagnostic imaging delivered by Dr Martin de Scally from South Africa. Dr Martin de Scally had a busy year delivering CE on a range of topics related to diagnostic imaging and infectious diseases in Botswana, Zimbabwe, Tanzania, Madagascar, and Ghana, several of which were in collaboration with Dr Lawson Cairns. Dr David Miller (Johannesburg, South Africa) lectured in Mozambique on internal medicine, and Dr Lawson Cairns used Clinician's Brief to deliver an innovative session at the same meeting. Dr Greg Irvine-Smith (Johannesburg, South Africa) spent 2 days in Kenya lecturing on surgery.

Delivering the CE program remains a team effort, and it would not be possible without the regional and local coordinators who donate hundreds of hours of their time to this incredible endeavour. Dr Lawson Cairns continues to do amazing work in Africa, organizing and overseeing a large number of meetings, often in challenging circumstances! The feedback from the lectures we provide remains outstanding and the engagement of our delegates in the meetings is evident. Our amazing speakers continue to deliver world class lectures in a whole range of settings, and I am incredibly grateful for their efforts.

Thank you again to the BSAVA for its ongoing support of this programme. Delivering CE in Africa is logistically difficult and expensive, but rewarding. The vets and students we work with are very keen to learn, as access to any educational resources about small animals remains very limited in most countries. We are starting to see the legacy of our actions in growing numbers of countries starting to organize their own fledgling CE programmes and small animal associations, and the expanding horizons of veterinary students. The BSAVA can be very proud of its role in facilitating these developments. IC

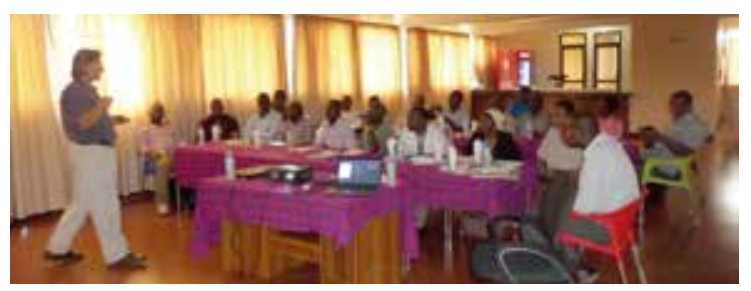

Dr Martin de Scally in Tanzania. 